\title{
Diverging Quantum Speed Limits: A Herald of Classicality
}

\author{
Pablo M. Poggi $\odot,{ }^{1,{ }^{*}}$ Steve Campbell $\odot,{ }^{2,3, \dagger}$ and Sebastian Deffner ${ }^{4,5, \$}$ \\ ${ }^{1}$ Center for Quantum Information and Control, Department of Physics and Astronomy, University of New Mexico, \\ Albuquerque, New Mexico 87131, USA \\ ${ }^{2}$ School of Physics, University College Dublin, Belfield, Dublin 4, Ireland \\ ${ }^{3}$ Centre for Quantum Engineering, Science, \& Technology, University College Dublin, Belfield, Dublin 4, Ireland \\ ${ }^{4}$ Department of Physics, University of Maryland, Baltimore County, Baltimore, Maryland 21250, USA \\ ${ }^{5}$ Instituto de Física 'Gleb Wataghin', Universidade Estadual de Campinas, Campinas, São Paulo 13083-859, \\ Brazil
}

(Received 21 July 2021; accepted 10 November 2021; published 9 December 2021)

\begin{abstract}
When is the quantum speed limit (QSL) really quantum? While vanishing QSL times often indicate emergent classical behavior, it is still not entirely understood what precise aspects of classicality are at the origin of this dynamical feature. Here, we show that vanishing QSL times (or, equivalently, diverging quantum speeds) can be traced back to reduced uncertainty in quantum observables and can thus be understood as a consequence of emerging classicality for these particular observables. We illustrate this mechanism by developing a QSL formalism for continuous-variable quantum systems undergoing general Gaussian dynamics. For these systems, we show that three typical scenarios leading to vanishing QSL times, namely large squeezing, small effective Planck's constant, and large particle number, can be fundamentally connected to each other. In contrast, by studying the dynamics of open quantum systems and mixed states, we show that the classicality that emerges due to incoherent mixing of states from the addition of classical noise typically increases the QSL time.
\end{abstract}

DOI: 10.1103/PRXQuantum.2.040349

\section{INTRODUCTION}

What distinguishes the classical world from the underlying quantum domain? Arguably the most prominent answers to this question revolve around the existence of uncertainty relations. While these relations have been tested, understood, and verified for pairs of canonical observables, as there is no observable for time, the uncertainty relation for energy and time remains harder to interpret. In its modern formulation the energy-time uncertainty relation is phrased as a quantum speed limit (QSL). In its original inception, the QSL reads [1-3] $\tau \geq \pi \hbar / 2 \Delta E \equiv \tau_{\mathrm{QSL}}$, where $\tau$ is the evolution time between orthogonal states, under a timeindependent Hamiltonian, $H$, and $\Delta E^{2}=\left\langle\psi\left|H^{2}\right| \psi\right\rangle-$ $\langle\psi|H| \psi\rangle^{2}$. QSLs have found widespread prominence

\footnotetext{
*ppoggi@unm.edu

†steve.campbell@ucd.ie

$\$$ deffner@umbc.edu
}

Published by the American Physical Society under the terms of the Creative Commons Attribution 4.0 International license. Further distribution of this work must maintain attribution to the author(s) and the published article's title, journal citation, and DOI. in, e.g., quantum information theory [4,5], while other formulations of QSLs provide fundamental and practical insight into the dynamics of complex systems [6-14]. Formally, QSLs can be elegantly expressed in terms of the geometry of quantum evolution [15-17], which in turn reveals a fundamental connection with the study of quantum parameter estimation $[18,19]$. In this geometric setting, QSLs have been generalized and applied to various scenarios of interest, notably open quantum systems [20-22] and quantum control [8,23-26].

Nevertheless, it is still debated what is really "quantum" about the QSL. Only recently, in two almost simultaneous works, Shanahan et al. [27] and Okuyama and Ohzeki [28] showed that bounds resembling the QSL also exist for classical dynamics. The origin of such speed limits, quantum as well as classical, rests in the notion of distinguishability of states. The speed limit is then a bound on the rate with which states become distinguishable from a previous configuration. While these results appear to put quantum and classical dynamics on an equal footing, some differences are expected to persist. The natural question, thus, has to be if and how a diverging quantum speed may be related to emergent classical behavior.

In this paper, we tackle this problem for a broad class of quantum systems, namely a collection of bosonic 
modes described by Gaussian Wigner functions under Gaussian-preserving dynamics [29,30]. These systems provide an ideal testbed to study QSLs for both quantum and classical systems and have widespread applications in continuous-variable (CV) quantum information [31]. In general, studying the QSL for CV systems is mathematically challenging due to their infinite-dimensional Hilbert spaces [32]. In contrast to previous work [27,33], here we do not work with a phase-space representation, but rather develop a QSL theory for Gaussian dynamics directly, which permits us to derive an expression for the QSL time in terms of finite-dimensional matrices using symplectic operators.

Using this formalism, we discuss three limits in which the QSL time vanishes: (i) $\hbar \rightarrow 0$, where $\hbar$ is interpreted as a parameter of the state, (ii) $r \rightarrow \infty$, where $r$ denotes the squeezing in the state, and (iii) $n \rightarrow \infty$, where $n$ is the number of modes. Thus, we establish that the emergent classicality linked to a vanishing QSL time can be associated with the reduced uncertainty in particular observables. For the special case of a single mode, we develop the theory further to show that, for each state, there exist Hamiltonians that maximize and minimize the QSL time. Finally, by applying our Gaussian QSL theory to general quantum evolution, we discuss the role of classical noise, mixed states, and nonunitary evolution. We illustrate how these aspects, which are related to a transition to classical behavior due to incoherent mixing of states rather than reduced uncertainty of observables, cannot decrease the QSL time.

\section{QUANTUM SPEED LIMIT FOR GAUSSIAN DYNAMICS}

We start by recalling the general formalism of geometric quantum speed limits. Consider a normalized distance between elements in the space of density operators given by $\Theta(\rho, \sigma)=2 \arccos \sqrt{F(\rho, \sigma)}$, where $F(\rho, \sigma)$ is a fidelity function satisfying $0 \leq F(\rho, \sigma) \leq 1$, and $F=1$ if and only if $\rho=\sigma$. Furthermore, consider general quantum dynamics given by $\rho_{t}=\Lambda_{t}\left[\rho_{0}\right]$, where $\left\{\Lambda_{t}, t \geq 0\right\}$ is a oneparameter family of completely positive trace-preserving maps. The quantum speed $V_{t}$ is computed by expanding the fidelity between the state $\rho_{t}$ and the state at a subsequent time $\rho_{t+d t}$,

$$
F\left(\rho_{t}, \rho_{t+d t}\right) \equiv 1-V_{t}^{2} d t^{2} \Longrightarrow d \Theta^{2}=4 V_{t}^{2} d t^{2} .
$$

Note that $V_{t}$ measures how fast quantum states become distinguishable from each other. Generally, $V_{t}$ is a function of $\rho_{t}$, but may also show an explicit dependence in time. Moreover, $V_{t}$ can be used to construct bounds on the evolution time in a variety of ways $[25,34,35]$, typically based on the relation

$$
\Theta\left(\rho_{0}, \rho_{\tau}\right) \leq 2 \int_{0}^{\tau} d t V_{t}
$$

Equation (2) expresses the fact that the distance between $\rho_{0}$ and $\rho_{\tau}$ must be smaller or equal to the length of the path taken by $\rho_{t}$ for $t \in[0, \tau]$.

For unitary dynamics and pure initial states, the natural choice is the quantum fidelity $F=\left|\left\langle\psi_{1} \mid \psi_{2}\right\rangle\right|^{2}$, for which $V_{t}=\Delta E_{t} / \hbar$ and Eq. (2) is the Anandan-Aharonov relation [15]. For time-independent Hamiltonians, the Mandelstam-Tamm bound can be easily inferred from Eq. (2); see also Ref. [36].

In the present analysis, we focus on the speed for Gaussian states and define the QSL time simply as its inverse, i.e., $\tau_{Q} \equiv V^{-1}$. The time dependence of $V$ has been dropped, since we can take $V$ to be the speed at the initial time $t=0$. It is straightforward to show (see Appendix A) that the other usual definitions of a QSL time are analogous to $\tau_{Q}$ in the asymptotic limit $\tau_{Q} \rightarrow 0$. Generic bosonic systems have a clear-cut classical limit, which makes them ideal to study the quantum-to-classical transition. Gaussian-preserving dynamics in systems of $n$ modes can be efficiently described using finite-dimensional operators corresponding to the symplectic group $\operatorname{Sp}(2 n)[30,37]$. These systems can be characterized by a vector of quadrature operators $\hat{\mathbf{z}}=\left(\hat{q}_{1}, \hat{p}_{1}, \ldots, \hat{q}_{n}, \hat{p}_{n}\right)$ with commutation relations [38]

$$
\left[\hat{z}_{k}, \hat{z}_{l}\right]=i \hbar \Omega_{k l}, \quad \text { where } \quad \Omega=\bigoplus_{j=1}^{n}\left(\begin{array}{cc}
0 & 1 \\
-1 & 0
\end{array}\right) .
$$

A state $\rho_{G}$ is Gaussian if its Wigner distribution is a Gaussian function in the quadrature variables. These states can be fully described by a $2 n$-dimensional real vector of expectation values $\mathbf{u}=\operatorname{tr}\left(\hat{\rho}_{G} \hat{\mathbf{z}}\right)$ and a real, symmetric $2 n \times 2 n$ covariance matrix $\Sigma=\operatorname{tr}\left(\hat{\rho}_{G}\left\{\delta \hat{\mathbf{z}}, \delta \hat{\mathbf{z}}^{T}\right\}\right)$, where $\delta \hat{\mathbf{z}} \equiv \hat{\mathbf{z}}-\mathbf{u}$, which is such that $\Sigma+i \hbar \Omega \geq 0$. Thus, the purity of $\rho_{G}$ is simply given by

$$
\operatorname{tr}\left\{\rho_{G}^{2}\right\}=\sqrt{\hbar / \operatorname{det}(\Sigma)} .
$$

For mathematical convenience, we now choose the metric $F$ to be the fidelity introduced in Refs. [39,40],

$$
F(\rho, \sigma)=\frac{\operatorname{tr}\{\rho \sigma\}}{\sqrt{\operatorname{tr}\left\{\rho^{2}\right\} \operatorname{tr}\left\{\sigma^{2}\right\}}} .
$$

Note that, for purity-preserving dynamics, Eq. (5) reduces to the relative purity [22] and the corresponding distance, $\Theta$, to that studied in Ref. [41]. For a CV system, $\rho$ is represented by an infinite-dimensional matrix; however, Gaussian states can be described by finite-dimensional 
objects $\rho \rightarrow(\Sigma, \mathbf{u})$. In terms of these, the fidelity of Eq. (5) can be expressed as $[42,43]$

$$
\begin{aligned}
F\left(\rho_{1}, \rho_{2}\right)= & \frac{\left[\operatorname{det}\left(\Sigma_{1}\right)\right]^{1 / 4}\left[\operatorname{det}\left(\Sigma_{2}\right)\right]^{1 / 4}}{\left\{\operatorname{det}\left[\left(\Sigma_{1}+\Sigma_{2}\right) / 2\right]\right\}^{1 / 2}} \\
& \times \exp \left[-\delta \mathbf{u}^{T}\left(\Sigma_{1}+\Sigma_{2}\right)^{-1} \delta \mathbf{u}\right],
\end{aligned}
$$

where $\delta \mathbf{u} \equiv \mathbf{u}_{2}-\mathbf{u}_{1}$. Consider now a transformation $\rho \rightarrow$ $\rho+d \rho$. If the evolution preserves the Gaussian character of the state, we can expand Eq. (6) to second order using $\Sigma \rightarrow \Sigma+d \Sigma$ and $\mathbf{u} \rightarrow \mathbf{u}+d \mathbf{u}$. This procedure, detailed in Appendix B, yields the expression

$$
\begin{aligned}
F(\rho, \rho+d \rho)= & 1-\frac{1}{16} \operatorname{tr}\left\{\left(\Sigma^{-1} d \Sigma\right)^{2}\right\} \\
& -\frac{1}{2} d \mathbf{u}^{T} \Sigma^{-1} d \mathbf{u},
\end{aligned}
$$

which we use to obtain explicit expressions for the quantum speed $V$ for different cases of interest.

We first focus on unitary evolutions generated by quadratic Hamiltonians of the form $H=\mathbf{z}^{T} G \mathbf{z} / 2$, with $G \in \mathbb{R}^{2 n \times 2 n}$ and symmetric. In this case the quadrature operators evolve according to $\mathbf{z}(t)=S(t) \mathbf{z}(0)$, with $S(t)$ a symplectic matrix (i.e., such that $S \Omega S^{T}=\Omega$ ) obeying $\dot{S}=\Omega G S(t)$, which in turn implies that initial Gaussian states remain Gaussian at all times. The unitary evolution of the covariance matrix and the displacement vector of a Gaussian state is given by

$$
d \Sigma=(\Omega G \Sigma-\Sigma G \Omega) d t \quad \text { and } \quad d \mathbf{u}=\Omega G \mathbf{u} d t .
$$

Inserting this into Eq. (7) and using Eq. (1), we can evaluate the quantum speed for unitary evolution of general (multimode, mixed) Gaussian states,

$$
\begin{aligned}
V_{U}^{2}= & \frac{1}{8}\left[-\operatorname{tr}\left\{G \Omega \Sigma^{-1} \Omega G \Sigma\right\}+\operatorname{tr}\left\{(\Omega G)^{2}\right\}\right] \\
& -\frac{1}{2} \mathbf{u}^{T} G \Omega \Sigma^{-1} \Omega G \mathbf{u} .
\end{aligned}
$$

This expression can be further simplified if $\rho \rightarrow\{\Sigma, \mathbf{u}\}$ is pure. In this case, using Williamson's theorem, it is straightforward to show that $-\Omega \Sigma^{-1} \Omega=\Sigma / \hbar^{2}$ (see Appendix C). This leads to the following general expression for the quantum speed for a generic pure Gaussian state undergoing Gaussian-preserving dynamics:

$$
\begin{aligned}
V_{U}^{2}= & \frac{1}{8 \hbar^{2}}\left[\operatorname{tr}\left\{(G \Sigma)^{2}\right\}+\hbar^{2} \operatorname{tr}\left\{(\Omega G)^{2}\right\}\right] \\
& +\frac{1}{2 \hbar^{2}} \mathbf{u}^{T} G \Sigma G \mathbf{u} .
\end{aligned}
$$

As expected from the Anandan-Aharonov relation, the expression in Eq. (9) coincides with the energy variance $\Delta E^{2} / \hbar^{2}$, a fact we prove by direct calculation in Appendix C. In the following, we turn our attention to analyzing the different limits in which the quantum speed diverges, leading to a vanishing QSL time.

\section{DIVERGING QUANTUM SPEED LIMITS}

\section{A. Limit of small Planck's constant}

We begin by analyzing the role of $\hbar$ in the QSL time. It is instructive to evaluate Eq. (10) for a generic multimode state evolving on a uniform Harmonic oscillator, where $G=\omega \mathbb{I}_{2 n \times 2 n}$. The covariance matrix of an arbitrary Gaussian pure state can be written as $\Sigma=\hbar O D O^{T}$, where $O$ is an orthogonal matrix and $D$ is a diagonal positive matrix of the form

$$
D=\bigoplus_{k=1}^{n}\left(\begin{array}{cc}
x_{k} & 0 \\
0 & 1 / x_{k}
\end{array}\right)
$$

The elements of $D$ describe the magnitude of the squeezing of the state and can be parametrized as $x_{k}=\exp \left(r_{k}\right)$, with $r_{k} \geq 0$. The resulting expression for the speed reads

$$
V_{U}^{2}=\frac{\omega^{2}}{8}\left(\operatorname{tr}\left\{D^{2}\right\}-2 n\right)+\frac{\omega^{2}}{2 \hbar} \mathbf{v}^{T} D \mathbf{v},
$$

where $\mathbf{v}=O^{T} \mathbf{u}$. Equation (12) exhibits two distinct contributions. The first term corresponds to the speed originating in the squeezing of the state (and it vanishes in its absence, i.e., when $x_{k}=1$ for all $k$ ); the second term indicates the displacement of the state.

Observe that the speed $V_{U}^{2}$ diverges as $\hbar \rightarrow 0$ [44]. This happens only for displaced states, since the first term is independent of $\hbar$. This behavior can be understood in terms of the evolved state becoming more distinguishable from the initial one as $\hbar$ is reduced. This is depicted for the single mode in Fig. 1(a). The necessary counterpart of $\hbar \rightarrow 0$ is that the state becomes more classical in the sense that the uncertainty in all quadratures is reduced. Thus, there is a straightforward connection between a vanishing QSL time and a reduced uncertainty associated with the state of the system. This result makes explicit the fact that the role of $\hbar$ in the QSL is precisely to set the minimum uncertainty, which limits the rate of change of the distinguishability. Since uncertainty can always be introduced in classical systems, a similar mechanism can be understood to lead to a speed limit for those systems [27,28].

\section{B. Limit of large squeezing}

We now turn to the second limit. For simplicity, we set without loss of generality $\mathbf{u}=0$, and therefore restrict to considering states that are centered at the origin in phase space. Using the same example as above, we have

$$
\operatorname{tr}\left\{D^{2}\right\}=2 \sum_{k=1}^{n} \cosh \left(2 r_{k}\right)=4 \sum_{k=1}^{n} \sinh ^{2}\left(r_{k}\right)+2 n,
$$



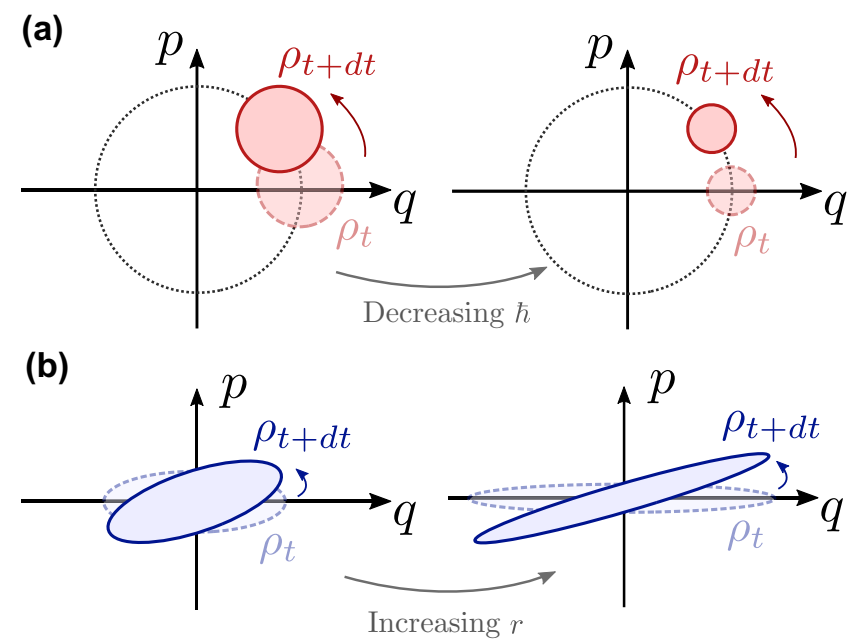

FIG. 1. Pure Gaussian states undergoing an infinitesimal evolution in phase space quickly become distinguishable if (a) $\hbar$, taken as a parameter of the state, is reduced, and (b) the squeezing parameter $r$ is increased.

which leads to

$$
V_{U}^{2}=\frac{\omega^{2}}{2} \sum_{k=1}^{n} \sinh ^{2}\left(r_{k}\right),
$$

revealing that, as the squeezing of the state increases, the quantum speed diverges. This phenomenon can again be rationalized from the fact that large squeezing allows for a faster increase in distinguishability, as schematically depicted in Fig. 1(b). Here we observe that a diverging speed is again associated with reduced uncertainty, in this case corresponding to the variance of the squeezed quadrature operator(s) of the state.

An important observation about the role of states and generators in the QSL follows from this example. Since the quantum speed is the rate at which the state of the system becomes distinguishable from its previous configuration under a given evolution, a vanishing QSL time can be achieved trivially if the generator (Hamiltonian in the unitary case) itself is unbounded [i.e., $\omega \rightarrow \infty$ in Eq. (12)]. What we have shown here is that a vanishing QSL time with a bounded generator is also possible, even in the case of a single-mode system $(n=1)$, provided the state is a highly squeezed state with $r \rightarrow \infty$. This is a feature of $\mathrm{CV}$ systems that is absent in the finite-dimensional case where the quantum speed is strictly upper bounded by the norm of the Hamiltonian $[26,45]$ and thus vanishing QSL times are prohibited (for fixed $\hbar$ ).

We investigate this behavior further by fully characterizing the quantum speed for generic, quadratic single-mode Hamiltonians. The complete derivation is relegated to Appendix D. For $n=1$, we can write a general mixed state as $\Sigma=\hbar c O D O^{T}$, where $O$ is a rotation matrix by an angle $\theta$, and $c>0$. The generator $G$ becomes

$$
G=g_{0} G_{0}+g_{S}\left[\sin (2 \phi) G_{1}+\cos (2 \phi) G_{2}\right],
$$

where $g_{0}, g_{S} \in \mathbb{R}$ are the weights corresponding to the number-preserving and number-nonpreserving parts of the generator; $G_{0}, G_{1}$, and $G_{2}$ are the $2 \times 2$ matrix representations of the single-mode Gaussian-preserving (quadratic) Hamiltonians $q^{2}+p^{2}, q^{2}-p^{2}$, and $q p+p q$, respectively. The angle $\phi$ is introduced to parametrize the relative contribution of each of the squeezing generators $G_{1}$ and $G_{2}$. In this case, $V_{U}$ can be evaluated exactly, yielding

$$
\begin{aligned}
V_{U}^{2}=\frac{1}{2}\{[ & \left.g_{0} \sinh (r)-g_{S} \sin (2 \delta) \cosh (r)\right]^{2} \\
& \left.+g_{S}^{2} \cos ^{2}(2 \delta)\right\},
\end{aligned}
$$

where we have introduced $\delta=\theta-\phi$.

The speed $V_{U}^{2}$ is plotted in Fig. 2 for various combinations of parameters. The maximum speed $V_{\max }^{2}$ occurs when $\delta=3 \pi / 4$. Introducing an overall energy scale $g$ such that $g_{0}=g \tilde{g_{0}}$ and $g_{S}=g \tilde{g_{S}}$, we have $V_{\max }^{2}=$ $g^{2} \cosh (2 r) / 2$, which grows as $\exp (2 r)$ for large $r$ and for $g_{0} / g_{S}=\tanh (r)$. For low squeezing, this amounts to setting $g_{S} \gg g_{0}$, while for high squeezing, it is achieved by $g_{S} \simeq g_{0}$. Consequently, for a single-mode system, there always exists a Hamiltonian for which the QSL time vanishes optimally as $\exp (-2 r)$ when the squeezing is large. It can also be shown that, for any degree of squeezing, an "opposite," minimum-speed Hamiltonian exists, for which $V_{U}$ is either zero or independent of $r$; see Appendix D.

\section{Limit of large system size}

Finally, we analyze the large $n$ limit. Taking $\mathbf{v}=$ $\left(\left\langle\tilde{q}_{1}\right\rangle,\left\langle\tilde{p}_{1}\right\rangle, \ldots,\left\langle\tilde{q}_{n}\right\rangle,\left\langle\tilde{p}_{n}\right\rangle\right)$, Eq. (12) becomes

$$
\begin{aligned}
V_{U}^{2}= & \frac{\omega^{2}}{2}\left[n\left(\frac{1}{n} \sum_{k=1}^{n} \sinh ^{2}\left(r_{k}\right)\right)+\frac{n}{\hbar}\left(\frac{1}{n} \sum_{k=1}^{n} \exp \left(r_{k}\right)\left\langle\tilde{q}_{k}\right\rangle^{2}\right.\right. \\
& \left.\left.+\frac{1}{n} \sum_{k=1}^{n} \exp \left(-r_{k}\right)\left\langle\tilde{p}_{k}\right\rangle^{2}\right)\right] .
\end{aligned}
$$

Assuming that the squeezing parameters $\left\{r_{k}\right\}$ and the displacements $\left\{\left\langle\tilde{q}_{k}\right\rangle,\left\langle\tilde{p}_{k}\right\rangle\right\}$ are independent of $n$, the quantities in the parentheses of Eq. (17) remain intensive as $n \rightarrow \infty$ and thus we find that $V_{U}^{2}$ diverges linearly with $n$. This behavior of $V_{U}$ bears close resemblance to the origin of the orthogonality catastrophe studied in Ref. [46].

Interestingly, the role of $n$ in the vanishing QSL time can be recast in terms of the two limits studied above. We begin with the first term in Eq. (17). For fixed $r_{k}$ 
(a)

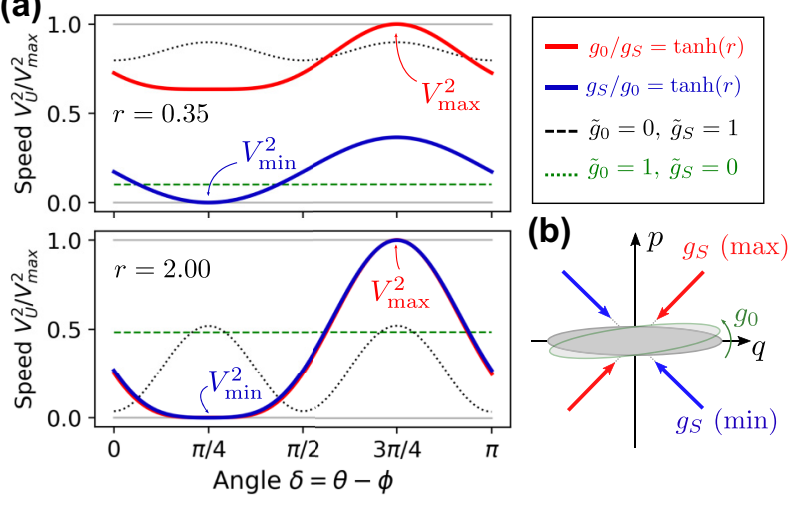

FIG. 2. Plots in (a) show $V_{U}^{2}$, normalized to its maximum value, as a function of $\delta=\theta-\phi$, where $\theta$ is the angle of the squeezed quadrature and $\phi$ is the angle characterizing the squeezing Hamiltonian; cf. Eq. (15). For a given degree of squeezing (top plot $r=0.35$, bottom plot $r=2.00$ ), the maximum speed is achieved at $\delta=3 \pi / 4$, when $g_{0} / g_{S}=\tanh (r)$, while the minimum is reached when $g_{S} / g_{0}=\tanh (r)$. These conditions give $g_{S} \simeq g_{0}$ for large $r$, as can be seen by the curves in the bottom plot. Other choices of $g_{0}, g_{S}$ are shown for comparison. (b) Schematic showing the action of the optimal Hamiltonians that maximize (red) and minimize (blue) the speed of a state squeezed along the $p$ quadrature. The arrows indicate the direction of squeezing.

values, the resulting multimode speed can be emulated by a single-mode system where the squeezing parameter $r$ obeys $\sinh ^{2}(r)=\sum_{k} \sinh ^{2}\left(r_{k}\right)$. For small $r_{k}=r^{(n)}$, we have $r \simeq \sqrt{n} r^{(n)}$, and, thus, in the absence of displacements, we find that the large- $n$ limit with finite squeezing is equivalent to the large squeezing limit of a single-mode evolution. We can include displacements in the analysis by now considering emulating the second term in Eq. (17) with a single-mode system. The components of the required displacement vector read

$$
\begin{aligned}
& \langle\tilde{q}\rangle=\frac{\exp (-r)}{n} \sum_{k=1}^{n} \exp \left(r_{k}\right)\left\langle\tilde{q}_{k}\right\rangle^{2}, \\
& \langle\tilde{p}\rangle=\frac{\exp (r)}{n} \sum_{k=1}^{n} \exp \left(-r_{k}\right)\left\langle\tilde{p}_{k}\right\rangle^{2} .
\end{aligned}
$$

In the absence of squeezing, $r_{k}=r=0$, the displacement vector length does not scale with $n$. Thus, the limit $n \rightarrow \infty$ is equivalent to letting $\hbar_{\mathrm{eff}} \equiv \hbar / n \rightarrow 0$, and thus it reduces to the first case considered above. In the presence of squeezing, the length of the displacement vector increases with $n$ since $\exp (r) \sim \exp (\sqrt{n})$. Thus, we can further normalize $\mathbf{v} \rightarrow \mathbf{v} / \cosh (r)$ such that $\|\mathbf{v}\|^{2} \sim \exp (r) / \cosh (r) \simeq 1$ as $n \rightarrow \infty$. Therefore, $\hbar_{\text {eff }} \equiv$ $\hbar /(n \cosh r) \sim \hbar / n^{2}$, which vanishes for $n \gg 1$.

\section{MIXED STATES AND NONUNITARY EVOLUTION}

So far we have analyzed the quantum speed of evolution for pure Gaussian states, and we have shown a relation between the diverging speed and a particular aspect of the classicality of the state, i.e., the uncertainty of an observable (or set of observables) vanishing. A seemingly separate notion of classicality is given by considering mixed states and purity-nonpreserving evolution. Mixed states are classical mixtures of pure states and the addition of classical noise is expected to reduce distinguishability [27,28].

To elucidate the matter, we now generalize our QSL theory of Gaussian states for general open quantum dynamics. Equation (7) can be applied to study any dynamics that preserves the Gaussian character of the state, such as general open diffusive dynamics [47]. Here we focus on the single-mode case $(n=1)$, which allows us to treat the most general Gaussian-preserving evolution in an exact way. The equations of motion can be written as (see Appendix E for further details)

$$
\begin{aligned}
\dot{\Sigma} & =\Omega G \Sigma-\Sigma G \Omega-g(\Sigma-M), \\
\dot{\mathbf{u}} & =(\Omega G-g / 2 \mathbb{I}) \mathbf{u},
\end{aligned}
$$

where $g \in \mathbb{R}$ and $M \in \mathbb{R}^{2 \times 2}$. As expected from Eq. (7), the quantum speed has the form

$$
V_{\mathrm{open}}^{2}=V_{\mathrm{cov}}^{2}+V_{\mathrm{mean}}^{2}
$$

with the first term stemming solely from the covariance matrix and the second term from the evolution of the mean values. Focusing on the former, we obtain $V_{\mathrm{cov}}^{2}=$ $V_{U}^{2}+\chi_{\mathrm{NU}}$, where

$$
V_{U}^{2}=\frac{1}{8}\left(\frac{1}{\eta^{2}} \operatorname{tr}\left\{(G \Sigma)^{2}\right\}+\operatorname{tr}\left\{(\Omega G)^{2}\right\}\right)
$$

is the contribution from unitary dynamics, i.e., the generalization of Eq. (9) for single-mode mixed states. Furthermore, we introduced $\eta=\sqrt{\operatorname{det}(\Sigma)}$, and

$$
\begin{aligned}
\chi_{\mathrm{NU}}= & \frac{g^{2}}{8}\left[1-\operatorname{tr}\left\{\Sigma^{-1} M\right\}+\operatorname{tr}\left\{\left(\Sigma^{-1} M\right)^{2}\right\} / 2\right] \\
& +\frac{g}{8 \eta^{2}} \operatorname{tr}\{\Sigma(G M \Omega-\Omega M G)\}
\end{aligned}
$$

is the contribution from the nonunitary part of the dynamics. For any choice of evolution given by $G, M$, and $g$, we can evaluate the speed for a squeezed thermal state $\Sigma=\eta\left(\beta_{s}\right) O D O^{T}$, where we have introduced the effective inverse temperature of the state $\beta_{s}$ via the usual parametrization $\eta\left(\beta_{s}\right)=2 \bar{n}_{s}+1 \equiv \operatorname{coth}\left(\beta_{s} \omega / 2\right)$. 
From Eq. (21), it becomes evident that $V_{U}^{2}$ is independent of $\beta_{s}$. The nonunitary contribution becomes

$$
\begin{aligned}
\chi_{\mathrm{NU}}=\frac{g^{2}}{8}\left[1-\frac{1}{\eta} \operatorname{tr}\left\{O D^{-1} O^{T} M\right\}\right. \\
\left.\quad+\frac{1}{g \eta} \operatorname{tr}\left\{O D O^{T}(G M \Omega-\Omega M G)\right\}\right]+\mathcal{O}\left(\eta^{-2}\right) .
\end{aligned}
$$

At large temperatures (small $\beta_{s}$ ), where classical noise dominates, we have $\eta \sim\left(\beta_{s} \omega\right)^{-1}$ and thus only the first term in $\chi_{\mathrm{NU}}$ survives. In this limit, the speed has an asymptotic, finite value $V_{\mathrm{cov}}^{2}=V_{U}^{2}+g^{2} / 8$, thus confirming that increasing classical noise always yields a nonzero speed limit time.

\section{A. Quantum Brownian motion}

As a last point, we explore the effects of the bath temperature on the quantum speed of evolution and compare its role with respect to the system's effective temperature. To this end, we focus on quantum Brownian motion (QBM), which describes the dynamics of a single harmonic oscillator interacting with a bosonic bath $[48,49]$. The master equation reads

$$
\begin{aligned}
\dot{\rho}= & -i\left[H_{0}, \rho\right]-\Delta[q,\{q, \rho\}] \\
& +\Pi[q,\{p, \rho\}]-i \gamma[q,\{p, \rho\}],
\end{aligned}
$$

where $H_{0}=\omega\left(q^{2}+p^{2}\right) / 2$. At high temperatures, the diffusion coefficients $\Delta$ and $\Pi$ can be written in terms of the damping rate $\gamma$ as $\Delta=\gamma / \beta_{B}+12 \gamma\left(\omega^{2}-\gamma^{2}\right) / \beta_{B}$ and $\Pi=-\gamma \beta_{B} / 12$, where $\beta_{B}$ is now the inverse temperature of the bosonic bath $[33,50]$. The dynamics of the QBM is Gaussian preserving [51,52] and can thus be cast in the form of Eq. (19) where $G=\omega \mathbb{I}, g=2 \gamma$, and

$$
M=\frac{1}{\gamma}\left(\begin{array}{cc}
\Delta & -\Pi / 2 \\
-\Pi / 2 & 0
\end{array}\right)
$$

To analyze the role of $\beta_{B}$ and $\beta_{S}$, we plot in Fig. 3 the QSL time $\tau=V_{\text {cov }}^{-1}$. In Fig. 3(a), $\tau$ is plotted for fixed bath temperature $\beta_{B}$ as a function of $\beta_{S}$ and for no squeezing, while in (b) the same is shown for the case of squeezing along $q$ quadrature, or squeezing along $p$ quadrature (dashed and dotted lines, respectively). As expected, we observe that the QSL time remains nonzero in all cases. Furthermore, $\tau$ reaches a bath-independent value at high temperature $\left(\beta_{S} \rightarrow 0\right)$, while it decays to a bath-dependent regime at low temperatures. In Figs. 3(c) and 3(d) the QSL time is plotted as a function of the inverse bath temperature for fixed values of $\beta_{B}$, and the same squeezing regimes as before. The resulting behavior is notably different, since $\tau$ vanishes at high bath temperature for all cases. The shape
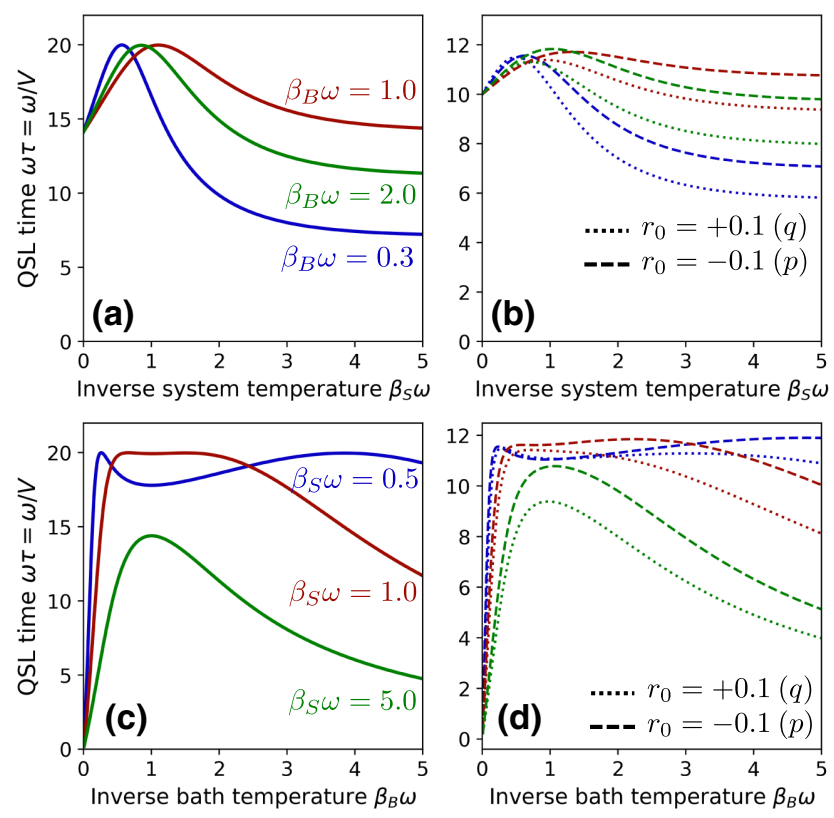

FIG. 3. Plots (a) and (b) depict the QSL time $\tau=V_{\text {cov }}^{-1}$ as a function of the inverse effective system temperature $\beta_{S}$ for different values of the bath temperature $\beta_{B}$. (a) $r=0$ (vacuum) and (b) $r= \pm 0.1$ (squeezing along $q$ or $p$ ). In these cases the QSL time is always nonzero. Plots (c) and (d) show the converse cases: $\tau=V_{\operatorname{cov}}^{-1}$ as a function of $\beta_{B}$ for fixed values of the system temperature. In all cases $\gamma=1$.

of these curves can be understood by analyzing the expression of the speed in the case with no squeezing $r=0$ (which leads to $V_{U}^{2}=0$ ). There, we obtain

$$
V_{\mathrm{QBM}}^{2}=\frac{\gamma^{2}}{2}\left(1-x\left(\beta_{S}, \beta_{B}\right)+\frac{x\left(\beta_{S}, \beta_{B}\right)^{2}}{2}\right)+\mathcal{O}\left(\beta_{B}\right),
$$

where $x\left(\beta_{s}, \beta_{B}\right)=\beta_{B}^{-1} \tanh \left(\beta_{S} \omega / 2\right)$. For fixed $\beta_{B}$, the speed remains bounded for all $\beta_{S}$, and reaches a bath-independent value of $\gamma^{2} / 4$ at large temperatures $\beta_{S} \ll 1$. This illustrates the behavior discussed above, where the system's effective temperature, related to the mixed nature of the state, cannot increase the speed arbitrarily and thus will not lead to vanishing QSL times. The role of the bath temperature is markedly different since, for fixed $\beta_{S}$, we find that $V_{\mathrm{QBM}}^{2}$ grows unboundedly as $T_{B}^{2}$. Note, however, that $T_{B}$ is a property of the generator of the nonunitary evolution, and throughout this work we have focused on vanishing QSL times for bounded generators.

Finally, other interesting features of the curves in Fig. 3 can be deduced from Eq. (26). First, note that the curves are not monotonic, and in particular the ones in (a) and (b) display a peak for a given combination of $\beta_{B}$ and $\beta_{S}$. This behavior is captured by Eq. (26) that predicts a minimum 
of the speed (maximum of QSL time) at $x\left(\beta_{S}, \beta_{B}\right)=1$. This condition corresponds to the case where the temperature of the system is roughly equal to the temperature of the bath (assumed to be large here). Under these conditions, the QBM dynamics has a steady state that is roughly equilibrated with the bath, and the closer the system state is to this equilibrium state, the smaller the speed. For arbitrary $\beta_{B}$, this same mechanism explains the emergence of the peaks, albeit the relation between the equilibrium temperature and the bath temperature is more intricate. On the other hand, Eq. (26) also predicts the plateau behavior seen at large $\beta_{S}$ in Figs. 3(a) and 3(b). For $x \rightarrow \infty$ (and in the regime of small $\beta_{B}$, where this expression is valid), we obtain a plateau value of $V_{\mathrm{QBM}}^{2} \simeq 1 /\left(2 \beta_{B}^{2}\right)$, which becomes larger as the bath temperature increases (and thus the state is further away from the equilibrium configuration).

\section{CONCLUDING REMARKS}

We have shown that vanishing QSL times in continuousvariable systems can be traced back to an underlying property: the asymptotically vanishing uncertainty of a set of particular observables that depend on the state and the dynamics. This result shows that a very particular notion of classicality, strictly related to this vanishing uncertainty, is responsible for the absence of a QSL. This property can emerge for systems as simple as a single bosonic mode in a highly squeezed state, but is absent in quantum systems with finite-dimensional Hilbert spaces. By studying the behavior of the QSL in open quantum systems, we have explored the behavior of other aspects of classicality on the QSL, and showed that, in contrast, the addition of classical noise, be it from considering mixed states, or from dissipative dynamics, will not lead to vanishing QSL times. To derive these results, we have developed a QSL framework for continuous-variable systems undergoing Gaussian-preserving dynamics. We expect this framework to have broader applications, particularly in the study of quantum control of $\mathrm{CV}$ systems and non-Markovianity $[53,54]$.

\section{ACKNOWLEDGMENTS}

This material is based upon work supported by the U.S. Department of Energy, Office of Science, National Quantum Information Science Research Centers, and Quantum Systems Accelerator. S.C. gratefully acknowledges the Science Foundation Ireland Starting Investigator Research Grant "SpeedDemon" (No. 18/SIRG/5508) for financial support. S.D. acknowledges support from the U.S. National Science Foundation under Grant No. DMR-2010127.
APPENDIX A: DIFFERENT DEFINITIONS OF THE QSL TIME

In the main text we define the QSL time as $\tau_{Q}=V^{-1}$; however, a more common definition is given by

$$
\tau_{1}(t)=\frac{\theta\left(\rho_{0}, \rho_{t}\right)}{(1 / t) \int_{0}^{t} d t^{\prime} V\left(t^{\prime}\right)}
$$

An alternative definition is given by $\tau_{2}(t)$, which is implicitly defined by the equation $[25,34]$

$$
\theta\left(\rho_{0}, \rho_{t}\right)=\int_{0}^{\tau_{2}(t)} d t^{\prime} V\left(t^{\prime}\right)
$$

In both cases, Eq. (2) ensures that $t \geq \tau_{i}$. For short $t, \tau_{i}$, one can approximate $V(t)$ as constant, i.e., evaluated at $\rho_{0}$, and thus from the expressions above one gets

$$
V(t) \tau_{i}=\theta\left(\rho_{0}, \rho_{t}\right) \rightarrow \tau_{i} \propto V^{-1}
$$

thus being proportional to $\tau_{Q}$, as intended.

\section{APPENDIX B: DERIVATION OF THE QUANTUM SPEED}

Here we derive Eq. (7) in the main text, which gives the expression for the quantum speed $V_{t}$ associated with the fidelity $F\left(\rho_{1}, \rho_{2}\right)$. First, take the Gaussian states $\rho_{1} \rightarrow$ $(\Sigma, \mathbf{u})$ and $\rho_{2} \rightarrow(\Sigma+d \Sigma, \mathbf{u}+d \mathbf{u})$. Using Eq. (6), we get

$$
\begin{aligned}
F\left(\rho_{1}, \rho_{2}\right)= & \frac{\operatorname{det}\left(\mathbb{I}+\Sigma^{-1} d \Sigma\right)^{1 / 4}}{\operatorname{det}\left(\mathbb{I}+\Sigma^{-1} d \Sigma / 2\right)^{1 / 2}} \\
& \times \exp \left[-d \mathbf{u}^{T}(2 \Sigma+d \Sigma)^{-1} d \mathbf{u}\right],
\end{aligned}
$$

where we exploit the fact that $\Sigma$ is invertible and properties of the determinant. Consider the first factor in the equation above, which is the ratio of two expressions of the form $\operatorname{det}(\mathbb{I}+\alpha B)^{\gamma}$. Any matrix $A$ obeys $\operatorname{det} A=$ $\exp \{\operatorname{tr}[\log (A)]\}$, and so one can expand the determinant to obtain

$$
\begin{aligned}
\operatorname{det}(\mathbb{I}+\alpha B)^{\gamma}= & \exp \left(\gamma \operatorname{tr}\left\{\alpha B-\frac{1}{2} \alpha^{2} B^{2}\right\}+\mathcal{O}\left(B^{3}\right)\right) \\
= & 1+\alpha \gamma \operatorname{tr}\{B\}-\frac{\gamma \alpha^{2}}{2} \operatorname{tr}\left\{B^{2}\right\} \\
& +\frac{\alpha^{2} \gamma^{2}}{2} \operatorname{tr}\{B\}^{2}+\mathcal{O}\left(B^{3}\right) .
\end{aligned}
$$

Using the general expression Eq. (B2), we can expand the first factor in Eq. (B1) in a straightforward way. The result 
reads

$$
\begin{aligned}
& \frac{\operatorname{det}\left(\mathbb{I}+\Sigma^{-1} d \Sigma\right)^{1 / 4}}{\operatorname{det}\left(\mathbb{I}+\Sigma^{-1} d \Sigma / 2\right)^{1 / 2}} \simeq 1-\frac{1}{16} \operatorname{tr}\left\{B^{2}\right\}, \quad \text { where } \\
& B=\Sigma^{-1} d \Sigma
\end{aligned}
$$

The second factor in Eq. (B1) is of the form $\exp \left(-d u^{T} L^{-1} d u\right)$, where $L=2 \Sigma+d \Sigma$. Expanding the inverse, one finds that up to terms that are quadratic in $(d \mathbf{u}, d \Sigma)$, the leading term corresponds to $L^{-1} \simeq \Sigma^{-1} / 2$. The resulting expansion reads

$$
\exp \left[-d \mathbf{u}^{T}(2 \Sigma+d \Sigma)^{-1} d \mathbf{u}\right] \simeq 1-\frac{1}{2} d \mathbf{u}^{T} \Sigma^{-1} d \mathbf{u} .
$$

Combining Eqs. (B3) and (B4) we get Eq. (7).

\section{APPENDIX C: QUANTUM SPEED FOR PURE STATES}

In order to derive Eq. (10) in the main text, we note that a general covariance matrix $\Sigma$ can be decomposed according to Williamson's theorem [30] as $\Sigma=\hbar S K S^{T}$, where $S \in$ $\mathrm{Sp}(2 n)$ and

$$
K=\bigoplus_{k=1}^{n}\left(\begin{array}{cc}
v_{k} & 0 \\
0 & v_{k}
\end{array}\right)
$$

The $\left\{v_{i}\right\}$ are the symplectic eigenvalues of $\Sigma$ such that the purity of the state is given by $\prod_{k} v_{k}$. A state is pure if and only if all $v_{k}=1$. In that case, $\Sigma=\hbar S S^{T}$ and, thus,

$$
\begin{aligned}
\Sigma^{-1} & =\frac{1}{\hbar}\left(S^{T}\right)^{-1} S^{-1} \Longrightarrow \Omega \Sigma^{-1} \Omega \\
& =-\frac{1}{\hbar} \Omega\left(S^{T}\right)^{-1} \Omega \Omega S^{-1} \Omega,
\end{aligned}
$$

where we have exploited the fact that $\Omega^{2}=-\mathbb{I}_{2 n \times 2 n}$. Now, recall that, by definition, a symplectic matrix $T$ is such that $T \Omega T^{T}=\Omega$. This condition can be rewritten as $\Omega T^{-1} \Omega=-T^{T}$. By evaluating $T=S$ and $T=S^{T}$, we then obtain $\Omega S^{-1} \Omega=-S^{T}$ and $\Omega\left(S^{T}\right)^{-1} \Omega=-S$. Then, Eq. (C2) reads

$$
\Omega \Sigma^{-1} \Omega=-\frac{1}{\hbar} S S^{T}=-\frac{1}{\hbar^{2}} \Sigma,
$$

which is used to derive Eq. (10) in the main text.

The Anandan-Aharonov relation [15] states that the speed of unitary evolution for pure states is given by $\Delta E^{2} / \hbar^{2}$, where $\Delta E^{2} \equiv\left\langle\hat{H}^{2}\right\rangle-\langle\hat{H}\rangle^{2}$. Thus, the expression for the speed in Eq. (10) has to be equal to this quantity. Here we check that this is indeed true by direct calculation.
First, recall that $\hat{H}=\frac{1}{2} \sum_{i j} G_{i j} \hat{z}_{i} \hat{z}_{j}$, and so

$$
\hat{H}-\langle\hat{H}\rangle=\frac{1}{2} \sum_{i j} G_{i j}\left(\hat{z}_{i} \hat{z}_{j}-\left\langle\hat{z}_{i} \hat{z}_{j}\right\rangle\right) .
$$

We can rewrite this expression in terms of the displaced quadrature operators $\delta \hat{\mathbf{z}}=\hat{\mathbf{z}}-\mathbf{u}$,

$$
\hat{H}-\langle\hat{H}\rangle=\frac{1}{2} \sum_{i j}\left(\delta \hat{z}_{i} \delta \hat{z}_{j}-\left\langle\delta \hat{z}_{i} \delta \hat{z}_{j}\right\rangle+u_{i} \delta \hat{z}_{j}+u_{j} \delta \hat{z}_{i}\right) .
$$

Then, we work out $\left\langle(\hat{H}-\langle\hat{H}\rangle)^{2}\right\rangle$, and use the fact that expectation values of even powers of the $\delta \hat{z}_{k}$ are zero, due to the Gaussian character of the state. The resulting expression reads

$$
\begin{aligned}
\left\langle(\hat{H}-\langle\hat{H}\rangle)^{2}\right\rangle= & \frac{1}{4} \sum_{i j k l} G_{i j} G_{k l}\left(\left\langle\delta \hat{z}_{i} \delta \hat{z}_{j} \delta \hat{z}_{k} \delta \hat{z}_{l}\right\rangle\right. \\
& -\left\langle\delta \hat{z}_{i} \delta \hat{z}_{j}\right\rangle\left\langle\delta \hat{z}_{k} \delta \hat{z}_{l}\right\rangle+u_{i} u_{k}\left\langle\delta \hat{z}_{j} \delta \hat{z}_{l}\right\rangle \\
& +u_{i} u_{l}\left\langle\delta \hat{z}_{j} \delta \hat{z}_{+}\right\rangle+u_{j} u_{k}\left\langle\delta \hat{z}_{i} \delta \hat{z}_{l}\right\rangle \\
& \left.+u_{j} u_{l}\left\langle\delta \hat{z}_{i} \delta \hat{z}_{k}\right\rangle\right)
\end{aligned}
$$

The next step is to use Wick's theorem in order to write the fourth-order moment in terms of the second-order moments,

$$
\begin{aligned}
& \left\langle\delta \hat{z}_{i} \delta \hat{z}_{j} \delta \hat{z}_{k} \delta \hat{z}_{l}\right\rangle=\left\langle\delta \hat{z}_{i} \delta \hat{z}_{j}\right\rangle\left\langle\delta \hat{z}_{k} \delta \hat{z}_{l}\right\rangle+\left\langle\delta \hat{z}_{i} \delta \hat{z}_{k}\right\rangle\left\langle\delta \hat{z}_{j} \delta \hat{z}_{l}\right\rangle \\
& \quad+\left\langle\delta \hat{z}_{i} \delta \hat{z}_{l}\right\rangle\left\langle\delta \hat{z}_{j} \delta \hat{z}_{k}\right\rangle
\end{aligned}
$$

and to note that

$$
\begin{aligned}
\delta \hat{z}_{i} \delta \hat{z}_{j}= & \frac{1}{2}\left(\left\{\delta \hat{z}_{i}, \delta \hat{z}_{j}\right\}+\left[\delta \hat{z}_{i}, \delta \hat{z}_{j}\right]\right) \rightarrow\left\langle\delta \hat{z}_{i} \delta \hat{z}_{j}\right\rangle \\
& =\frac{1}{2}\left(\Sigma_{i j}+i \hbar \Omega_{i j}\right) \equiv \frac{1}{2} V_{i j} .
\end{aligned}
$$

With these elements in place, we now can combine Eq. (C6) with Eqs. (C7) and (C8). The resulting expression reads

$$
\begin{aligned}
\Delta E^{2}= & \frac{1}{16} \sum_{i j k l} G_{i j} G_{k l}\left(V_{i k} V_{j l}+V_{i l} V_{j k}\right) \\
& +\frac{1}{8} \sum_{i j k l} G_{i j} G_{k l}\left(u_{i} u_{k} V_{j l}\right. \\
& \left.+u_{i} u_{l} V_{j k}+u_{j} u_{k} V_{i l}+u_{j} u_{l} V_{i k}\right) \\
= & \frac{1}{8} \operatorname{tr}\left[G V G V^{T}\right]+\frac{1}{2} \mathbf{u}^{T} G V G \mathbf{u} \\
= & \frac{1}{8}\left\{\operatorname{tr}\left[(G \Sigma)^{2}\right]+\hbar^{2} \operatorname{tr}\left[(G \Omega)^{2}\right]\right\}+\frac{1}{2} \mathbf{u}^{T} G \Sigma G \mathbf{u},
\end{aligned}
$$

where we have used the fact that $\mathbf{u}^{T} G \Omega G \mathbf{u}=0$ for all $\mathbf{u}$ since $G \Omega G$ is an antisymmetric matrix. 


\section{APPENDIX D: QSL FOR SINGLE-MODE GAUSSIAN UNITARY EVOLUTION}

For $n=1$, the most general covariance matrix can be written as

$$
\Sigma=\hbar c R(\theta) D R(\theta)^{T} \quad \text { with } \quad R(\theta)=\left(\begin{array}{cc}
\cos \theta & \sin \theta \\
-\sin \theta & \cos \theta
\end{array}\right)
$$

and $D=\operatorname{diag}[\exp (r), \exp (-r)]$. For this analysis, we focus on the role of squeezing, and we thus consider undisplaced states $(\mathbf{u}=0)$. The Hamiltonian generating the evolution is an element of the algebra $\mathfrak{s p}(2)$, which has dimension $2 n-1=3$ and is spanned by the elements

$$
G_{0}=\left(\begin{array}{ll}
1 & 0 \\
0 & 1
\end{array}\right), \quad G_{1}=\left(\begin{array}{cc}
1 & 0 \\
0 & -1
\end{array}\right), \quad G_{2}=\left(\begin{array}{ll}
0 & 1 \\
1 & 0
\end{array}\right) .
$$

So, we consider dynamics driven by the most general generator

$$
G=g_{0} G_{0}+g_{1} G 1+g_{2} G_{2} \equiv g_{0} G_{0}+g_{S} G_{S}(\phi),
$$

where we have introduced the alternative parametrization $G_{S}(\phi)=R(\phi) G_{2} R(\phi)^{T}, g_{1}=g_{s} \sin (2 \phi)$, and $g_{2}=$ $g_{s} \cos (2 \phi)$. In this notation, $g_{0}$ is the weight of the numberpreserving part of the Hamiltonian, while $g_{S}$ is the weight of the number-nonpreserving part of $G$. Using the expressions in Eqs. (D1) and (D3), we can evaluate the speed in Eq. (9). After some algebraic manipulation, the result reads

$$
V_{U}^{2}=\frac{1}{2}\left\{\left[g_{0} \sinh (r)-g_{S} \sin (2 \delta) \cosh (r)\right]^{2}+g_{S}^{2} \cos ^{2}(2 \delta)\right\},
$$

where we have introduced $\delta=\theta-\phi$.

Our goal is to derive, for a given value of squeezing, the Hamiltonian that maximizes and minimizes the speed. Differentiating Eq. (D4) with respect to $\delta$ and equating it to zero reveals the existence, in the interval $\delta \in[0, \pi]$, of the extrema

$$
\delta_{c}^{(1)}=\frac{\pi}{4} \quad \text { and } \quad \delta_{c}^{(2)}=\frac{3 \pi}{4}
$$

for all values of parameters, while two extra extrema $\delta_{c}^{( \pm)}$ appear if $g_{0} / g_{S} \leq \tanh (r)$, which obey the equation

$$
\sin \left(2 \delta_{c}^{( \pm)}\right)=\frac{g_{0}}{g_{S}} \operatorname{coth}(r) .
$$

Straightforward stability analysis reveals that $\delta_{c}^{(2)}$ is always a maximum (and furthermore, it is global), the $\delta_{c}^{( \pm)}$are always minima (when they exist), and thus $\delta_{c}^{(1)}$ is a minima for $g_{0} / g_{S}>\tanh (r)$ and a maxima otherwise. The resulting situation is depicted in Fig. 2. by

Let us now discuss the maximum speed, which is given

$$
V_{\max }^{2}=V_{U}^{2}\left(\delta_{c}^{(2)}\right)=\frac{1}{2}\left[g_{0} \sinh (r)+g_{S} \cosh (r)\right]^{2} .
$$

For a fixed degree of squeezing $r$, the above expression reaches its maximum value $g^{2} \cosh (2 r) / 2$ when $g_{0} / g_{S}=$ $\tanh (r)$. Here $g$ is taken to be some overall Hamiltonian strength that we assume to be fixed, i.e., $g_{0}=g \tilde{g_{0}}$ and $g_{S}=g \tilde{g_{s}}$. This result tells us that we can always achieve a maximum speed proportional to $\cosh (2 r) \sim \exp (2 r)$ by using an optimal choice Hamiltonian. If $r$ is small, the choice is to set $g_{S} \gg g_{0}$. For large $r$, on the other hand, the best choice is to set $g_{S} \simeq g_{0}$.

Conversely, one can analyze the minimum possible speed $V_{\min }^{2}$ for these systems. In this case, the expression for $V_{\min }^{2}$ is different depending on the relation between $g_{0}$ and $g_{S}$. If $g_{0}>g_{S} \tanh (r)$ then the minimum occurs at $\delta_{c}^{(1)}$ and we obtain

$$
V_{\min }^{2}=V_{U}^{2}\left(\delta_{c}^{(1)}\right)=\frac{1}{2}\left[g_{0} \sinh (r)-g_{S} \cosh (r)\right]^{2} .
$$

This is the naturally opposite situation to the one described before. The minimum possible speed is 0 , and its achieved for $g_{S} / g_{0}=\tanh (r)$; for low squeezing, the optimal choice is one where $g_{0} \gg g_{S}$. At higher squeezing, the optimal choice is $g_{0} \simeq g_{S}$ as before. If $g_{0}<g_{S} \tanh (r)$ then the minimum occurs at both $\delta_{c}^{( \pm)}$, for which

$$
V_{\min }^{2}=V_{U}^{2}\left(\delta_{c}^{( \pm)}\right)=\frac{1}{2}\left(g_{S}^{2}-g_{0}^{2}\right) .
$$

Interestingly, even when the speed cannot be turned to zero in this parameter regime, it will always be independent of the amount of squeezing in the system.

\section{APPENDIX E: QUANTUM SPEED FOR OPEN SYSTEM DYNAMICS}

Markovian Gaussian-preserving evolution can be shown to lead to the following general equations of motion for a $n$-mode system [47]:

$$
\begin{aligned}
\dot{\Sigma} & =B \Sigma+\Sigma B^{T}+D, \\
\dot{\mathbf{u}} & =B \mathbf{u} .
\end{aligned}
$$

Here $B$ and $D$ are generic $2 n \times 2 n$ matrices that obey the relation $D+i \Omega B_{a} \Omega^{T} \geq 0$, where $B_{a}=\Omega^{T} B-B^{T} \Omega$. It is convenient to write $B=\Omega(G+F)$, where $G$ is symmetric (i.e., corresponding to the unitary dynamics, $\Omega G=A$ ) and $F$ is antisymmetric. For a single-mode system, $n=1$, expressions simplify considerably since the most general antisymmetric matrix can be written as

$$
F=\left(\begin{array}{cc}
0 & g / 2 \\
-g / 2 & 0
\end{array}\right) .
$$

Then, the generator takes the form $B=\Omega(G+F)=$ $A+\Omega F=A-g \mathbb{I} / 2$. By defining $M=D / g$, this proves 
Eq. (19) in the main text. Note that the condition over $D$ reads $\operatorname{det} D \geq \operatorname{det}(2 F)=g^{2}$ and so $\operatorname{det} M \geq 1$.

The quantum speed arising from the evolution of the covariance matrix is

$$
V_{\mathrm{cov}}^{2}=\frac{1}{16} \operatorname{tr}\left\{\left(\Sigma^{-1} \dot{\Sigma}\right)^{2}\right\}
$$

Since $\dot{\Sigma}$ is the sum of a unitary and a nonunitary contribution, and given the quadratic dependence of the speed, naturally one obtains $V_{\mathrm{cov}}^{2}=V_{U}^{2}+\chi_{\mathrm{NU}}$, i.e., a contribution solely from unitary dynamics plus a nonunitary correction, which itself can be thought of as a combination of a purely nonunitary and a cross term.

[1] L. Mandelstam and I. Tamm, The uncertainty relation between energy and time in non-relativistic quantum mechanics, J. Phys. USSR 9, 249 (1945).

[2] G. N. Fleming, A unitarity bound on the evolution of nonstationary states, Il Nuovo Cimento A (1965-1970) 16, 232 (1973).

[3] K. Bhattacharyya, Quantum decay and the MandelstamTamm-energy inequality, J. Phys. A: Math. Gen. 16, 2993 (1983).

[4] V. Giovannetti, S. Lloyd, and L. Maccone, Quantum limits to dynamical evolution, Phys. Rev. A 67, 052109 (2003).

[5] L. B. Levitin and T. Toffoli, Fundamental Limit on the Rate of Quantum Dynamics: The Unified Bound is Tight, Phys. Rev. Lett. 103, 160502 (2009).

[6] S. Deffner and S. Campbell, Quantum speed limits: From Heisenberg's uncertainty principle to optimal quantum control, J. Phys. A: Math. Theor. 50, 453001 (2017).

[7] M. R. Frey, Quantum speed limits - primer, perspectives, and potential future directions, Quantum Inf. Process 15, 3919 (2016).

[8] T. Caneva, M. Murphy, T. Calarco, R. Fazio, S. Montangero, V. Giovannetti, and G. E. Santoro, Optimal Control at the Quantum Speed Limit, Phys. Rev. Lett. 103, 240501 (2009).

[9] C. Arenz, B. Russell, D. Burgarth, and H. Rabitz, The roles of drift and control field constraints upon quantum control speed limits, New J. Phys. 19, 103015 (2017).

[10] P. M. Poggi, Geometric quantum speed limits and shorttime accessibility to unitary operations, Phys. Rev. A 99, 042116 (2019).

[11] M. R. Lam, N. Peter, T. Groh, W. Alt, C. Robens, D. Meschede, A. Negretti, S. Montangero, T. Calarco, and A. Alberti, Demonstration of Quantum Brachistochrones between Distant States of an Atom, Phys. Rev. X 11, 011035 (2021).

[12] G. Ness, M. R. Lam, W. Alt, D. Meschede, Y. Sagi, and A. Alberti, Observing quantum-speed-limit crossover with matter wave interferometry, ArXiv:2104.05638 (2021).

[13] A. del Campo, Probing Quantum Speed Limits with Ultracold Gases, Phys. Rev. Lett. 126, 180603 (2021).

[14] R. Puebla, S. Deffner, and S. Campbell, Kibble-Zurek scaling in quantum speed limits for shortcuts to adiabaticity, Phys. Rev. Res. 2, 032020 (2020).
[15] J. Anandan and Y. Aharonov, Geometry of Quantum Evolution, Phys. Rev. Lett. 65, 1697 (1990).

[16] A. K. Pati, New derivation of the geometric phase, Phys. Lett. A 202, 40 (1995).

[17] D. P. Pires, M. Cianciaruso, L. C. Céleri, G. Adesso, and D. O. Soares-Pinto, Generalized Geometric Quantum Speed Limits, Phys. Rev. X 6, 021031 (2016).

[18] S. L. Braunstein and C. M. Caves, Statistical Distance and the Geometry of Quantum States, Phys. Rev. Lett. 72, 3439 (1994).

[19] V. Giovannetti, S. Lloyd, and L. Maccone, Quantum Metrology, Phys. Rev. Lett. 96, 010401 (2006).

[20] M. M. Taddei, B. M. Escher, L. Davidovich, and R. L. de Matos Filho, Quantum Speed Limit for Physical Processes, Phys. Rev. Lett. 110, 050402 (2013).

[21] S. Deffner and E. Lutz, Quantum Speed Limit for NonMarkovian Dynamics, Phys. Rev. Lett. 111, 010402 (2013).

[22] A. del Campo, I. L. Egusquiza, M. B. Plenio, and S. F. Huelga, Quantum Speed Limits in Open System Dynamics, Phys. Rev. Lett. 110, 050403 (2013).

[23] M. H. Goerz, T. Calarco, and C. P. Koch, The quantum speed limit of optimal controlled phasegates for trapped neutral atoms, J. Phys. B 44, 154011 (2011).

[24] G. C. Hegerfeldt, Driving at the Quantum Speed Limit: Optimal Control of a Two-Level System, Phys. Rev. Lett. 111, 260501 (2013).

[25] P. M. Poggi, F. C. Lombardo, and D. A. Wisniacki, Quantum speed limit and optimal evolution time in a two-level system, Europhys. Lett. 104, 40005 (2013).

[26] P. Poggi, Analysis of lower bounds for quantum control times and their relation to the quantum speed limit, Anales AFA 31, 29 (2020).

[27] B. Shanahan, A. Chenu, N. Margolus, and A. del Campo, Quantum Speed Limits across the Quantum-To-Classical Transition, Phys. Rev. Lett. 120, 070401 (2018).

[28] M. Okuyama and M. Ohzeki, Quantum Speed Limit is not Quantum, Phys. Rev. Lett. 120, 070402 (2018).

[29] A. Ferraro, S. Olivares, and M. G. A. Paris, Gaussian states in continuous variable quantum information, ArXiv:quant-ph:0503237 (2005).

[30] G. Adesso, S. Ragy, and A. R. Lee, Continuous variable quantum information: Gaussian states and beyond, Open Syst. Inf. Dyn. 21, 1440001 (2014).

[31] C. Weedbrook, S. Pirandola, R. García-Patrón, N. J. Cerf, T. C. Ralph, J. H. Shapiro, and S. Lloyd, Gaussian quantum information, Rev. Mod. Phys. 84, 621 (2012).

[32] P. Marian and T. A. Marian, Quantum speed of evolution in a markovian bosonic environment, Phys. Rev. A 103, 022221 (2021).

[33] S. Deffner, Geometric quantum speed limits: A case for Wigner phase space, New J. Phys. 19, 103018 (2017).

[34] N. Mirkin, F. Toscano, and D. A. Wisniacki, Quantumspeed-limit bounds in an open quantum evolution, Phys. Rev. A 94, 052125 (2016).

[35] E. O'Connor, G. Guarnieri, and S. Campbell, Action quantum speed limits, Phys. Rev. A 103, 022210 (2021).

[36] S. Deffner and E. Lutz, Energy-time uncertainty relation for driven quantum systems, J. Phys. A: Math. Theor. 46, 335302 (2013).

[37] B. Hall, Lie Groups, Lie Algebras, and Representations: an Elementary Introduction Vol. 222 (Springer, New York, 2015). 
[38] Since we are interested in studying the role of $\hbar$ in the QSL, we have defined the quadrature operators to be independent of $\hbar$. For the typical harmonic oscillator Hamiltonian, $H_{\mathrm{HO}}=P^{2} / 2 m+m \omega^{2} / 2 Q^{2}$, the definition used in the main text corresponds to taking $q=\sqrt{m \omega} Q$ and $p=P / \sqrt{m \omega}$ such that $[q, p]=[Q, P]=i \hbar$.

[39] X. Wang, C.-S. Yu, and X. X. Yi, An alternative quantum fidelity for mixed states of qudits, Phys. Lett. A 373, 58 (2008).

[40] Z. Sun, J. Liu, J. Ma, and X. Wang, Quantum speed limits in open systems: Non-Markovian dynamics without rotatingwave approximation, Sci. Rep. 5, 8444 (2015).

[41] F. Campaioli, F. A. Pollock, F. C. Binder, and K. Modi, Tightening Quantum Speed Limits for Almost all States, Phys. Rev. Lett. 120, 060409 (2018).

[42] P. Marian and T. A. Marian, Uhlmann fidelity between twomode Gaussian states, Phys. Rev. A 86, 022340 (2012).

[43] V. Link and W. T. Strunz, Geometry of Gaussian quantum states, J. Phys. A: Math. Theor. 48, 275301 (2015).

[44] K. Bolonek-Lasoń, J. Gonera, and P. Kosiński, Classical and quantum speed limits, Quantum 5, 482 (2021).

[45] D. C. Brody, G. W. Gibbons, and D. M. Meier, Timeoptimal navigation through quantum wind, New J. Phys. 17, 033048 (2015).

[46] T. Fogarty, S. Deffner, T. Busch, and S. Campbell, Orthogonality Catastrophe as a Consequence of the Quantum Speed Limit, Phys. Rev. Lett. 124, 110601 (2020).
[47] M. G. Genoni, L. Lami, and A. Serafini, Conditional and unconditional Gaussian quantum dynamics, Contemp. Phys. 57, 331 (2016).

[48] B. L. Hu, J. P. Paz, and Y. Zhang, Quantum Brownian motion in a general environment: Exact master equation with nonlocal dissipation and colored noise, Phys. Rev. D 45, 2843 (1992).

[49] M. A. Schlosshauer, Decoherence: and the Quantum-ToClassical Transition (Springer Science \& Business Media, New York, 2007).

[50] S. Deffner, Quantum entropy production in phase space, Europhys. Lett. 103, 30001 (2013).

[51] R. Vasile, S. Olivares, M. G. A. Paris, and S. Maniscalco, Continuous-variable-entanglement dynamics in structured reservoirs, Phys. Rev. A 80, 062324 (2009).

[52] G. Torre and F. Illuminati, Exact non-Markovian dynamics of Gaussian quantum channels: Finite-time and asymptotic regimes, Phys. Rev. A 98, 012124 (2018).

[53] R. Wu, R. Chakrabarti, and H. Rabitz, Optimal control theory for continuous-variable quantum gates, Phys. Rev. A 77, 052303 (2008).

[54] H. R. Jahromi, K. Mahdavipour, M. Khazaei Shadfar, and R. Lo Franco, Witnessing non-Markovian effects of quantum processes through Hilbert-Schmidt speed, Phys. Rev. A 102, 022221 (2020). 